\title{
TURISMO, NATURALEZA Y ACCESIBILIDAD: USO DEL ÁREA NACIONAL DE RECREACIÓN SANTAY PARA PERSONAS CON DISCAPACIDAD FÍSICA
}

\section{TOURISM, NATURE AND ACCESSIBILITY: USE OF THE NATIONAL SANTAY RECREATION AREA FOR PEOPLE WITH PHYSICAL DISABILITIES}

\author{
César Augusto Santana Moncayo, Mgs. \\ Magíster en Docencia y Gerencia de Educación Superior (Ecuador). \\ Docente Titular de la Facultad de Turismo y Hotelería de \\ la Universidad Tecnológica ECOTEC, Ecuador. \\ csantana@ecotec.edu.ec
}

\section{ARTÍCULO DE INVESTIGACIÓN}

Recibido: 2 de septiembre de 2019.

Aceptado: 28 de octubre de 2019.

\section{RESUMEN}

El presente artículo es un breve estudio de caso relacionado con la visita de un grupo de once personas con discapacidad física hacia el Área Nacional de Recreación Islas Santay y Gallo, pero enfocándose en la primera isla. El escrito empieza con una breve descripción de la Isla Santay, desde el punto de vista geográfico y morfológico, contando luego un poco de su historia, hasta presentar su actual oferta relacionada con el turismo y la ecología, para luego presentar un estudio de caso relacionado al turismo accesible para personas con discapacidad. Finalmente, se relatan las conclusiones a las que se ha llegado luego del análisis de los datos obtenidos, para terminar con conclusiones basadas en la experiencia relatada.

Palabras clave: Turismo, accesibilidad, comunidad, Isla, Santay, Guayaquil, Durán 


\section{ABSTRACT}

This document is a brief case study related to the visit of a group of eleven (11) people with physical disabilities to the National Recreation Area Santay and Gallo Islands, but focusing on the first island

The paper begins with a brief description of Santay Island, from the geographical and morphological point of view, then telling a bit of its history, until presenting its current offer related to tourism and ecology, and then presenting a related case study Accessible tourism for people with disabilities. Finally, the conclusions reached after the analysis of the data obtained are related, to conclude with conclusions based on the experience reported.

Keywords: Tourism, accesibility, community, island, Santay, Guayaquil, Duran.

\section{INTRODUCCIÓN}

A través de esta ponencia, se busca definir la forma en como la accesibilidad es percibida y trabajada en la Isla Santay, a través del turismo comunitario, sustentable y ecológico que se desarrolla en la misma. El objetivo general de esta presentación es calificar los aspectos de accesibilidad en este espacio natural, a través de una breve encuesta realizada a un grupo de personas con discapacidad física, usuarios turísticos, al menos por una ocasión, de la Isla Santay.

Como objetivos específicos se destacan: a) relatar, en forma breve, la historia de la Isla Santay, desde sus primeros pobladores hasta la actualidad, a través de la búsqueda de información secundaria, principalmente de artículos científicos variados; y, b) Explicar las razones por las que la accesibilidad en el área natural descrita debe ser considerada como parte de su calidad, a fin de convertirse en un espacio adecuado para demostrar la accesibilidad universal.

Como resultado principal, se obtuvieron respuestas relacionadas con la seguridad y comodidad de utilizar el sitio turístico, su acceso, calidad de información y servicios complementarios dentro de la Isla, las mismas que luego se redactaron dentro de las conclusiones del trabajo. 


\section{REVISIÓN TEÓRICA}

a) El turismo sostenible, el desarrollo sostenible y la Isla Santay

El turismo sostenible y su vinculación con el desarrollo local de diferentes sectores humanos, sobre todo rurales, se puede identificar en una gran variedad de lugares y proyectos que, a día de hoy, permiten el sustento y mejora del nivel de vida de una gran cantidad de comunidades, tanto alrededor del mundo como en Ecuador.

Para poder comprender a cabalidad el concepto de turismo sostenible es necesario revisar, en primer lugar, el concepto de desarrollo sostenible. Fue en el informe "Nuestro futuro común", llamado también "Informe Bruntland", donde se lo definió, por primera vez, de la siguiente manera: “...es el desarrollo que satisface las necesidades de la generación presente sin comprometer la capacidad de las generaciones futuras para satisfacer sus propias necesidades". (Comisión Mundial sobre el Medio Ambiente y Desarrollo, 1987). A pesar de lo acertado del concepto, muchos científicos y pensadores han opinado, desde que surgió, que tanto la idea de desarrollo, como la sostenibilidad, no son compatibles: la primera se dirige hacia el crecimiento sin fin, mientras que la segunda trata de mejorar las condiciones del planeta y sus recursos (Gómez, 2014).

El concepto de desarrollo turístico sostenible, que se basa en lo que indica la Organización Mundial del Turismo, a partir del Informe Bruntland mencionado en párrafos anteriores, quedó definido de la siguiente manera:

(...) es el que atiende a las necesidades de los turistas actuales y de las regiones receptoras y al mismo tiempo protege y fomenta las oportunidades para el futuro. Se concibe como una vía hacia la gestión de todos los recursos de forma que puedan satisfacerse las necesidades económicas, sociales y estéticas, respetando al mismo tiempo la integridad cultural, los procesos ecológicos esenciales, la diversidad biológica y los sistemas que sostienen la vida (Organización Mundial del Turismo, 2000).

Con ambos conceptos en mente, se puede entonces realizar los diferentes ejercicios de comprensión sobre las actuaciones turísticas que existen en la Isla Santay, un espacio natural 
ubicado entre Guayaquil y Durán (provincia del Guayas, Ecuador), e interpretar si lo que acontece en este lugar, puede catalogarse como sostenible.

Se debe entender, entonces, que el turismo que se desarrolla en la Isla Santay puede ser interpretado en dos aspectos fundamentales: por un lado, como una referencia de importancia ecológica, tanto para la ciudad de Guayaquil como al cantón Durán; y, por otro lado, debe ser observado como un espacio adecuado para el desarrollo de un turismo de disfrute de la naturaleza, ya que cuenta con condiciones adecuadas para atravesar el espacio y permitir su disfrute.

Sin embargo, es necesario también analizar el sitio desde una perspectiva diferente: la de las personas con discapacidad. En efecto, es bien sabido que el turismo puede ser (de hecho, lo es), una fuente de trabajo e ingreso para personas con y sin discapacidad (Jurado, 2014), ya que no solo permite la actividad laboral y una remuneración justa, sino que permite acrecentar el conocimiento de los involucrados, lo que redunda, por supuesto, en una mejora de servicio.

\section{Breve historia de la Isla Santay}

La Isla Santay se encuentra ubicada en “...el curso del río Guayas, límite natural de los cantones Guayaquil y Durán, en la provincia del Guayas, a 800 metros de distancia de la ciudad de Guayaquil y pertenece a la jurisdicción del cantón Durán" (Hidalgo, Villafuerte, y Ortiz, 2015). De acuerdo a datos del censo poblacional del 2010, la Isla cuenta con 226 habitantes, con una tasa de crecimiento de $1,3 \%$ anual. De esta población, el $32 \%$ corresponde a hombres adultos, el $23 \%$ mujeres adultas, $10 \%$ de jóvenes y $35 \%$ de niños. De todos ellos, el $58 \%$ correspondía a la población económicamente activa, con un $53 \%$ de la población que, en la actualidad, trabaja en actividades de turismo comunitario (Pérez, Díaz, Muñoz, y Ortega, 2019).

En la isla, hasta el año 1980, las familias que la habitaban se dedicaban a la ganadería y al cultivo de arroz, ya que posee planicies inundables, típicas de la llanura de inundación de la cuenca baja del río Guayas (Alcívar y Freire, 2017). Se sabe que la isla empezó a poblarse en el siglo XIX, y, por las características del suelo, es que se realizaban las actividades agropecuarias antes mencionadas (Ordoñez, 2017). 
En los años 70's, en la Isla existían siete haciendas ubicadas en diferentes parcelas, y con la administración de cada una en forma independiente. Los nombres de los propietarios de aquellas haciendas fueron: (Perrone, 2013).

Tabla 1. Propietarios de las haciendas existentes en la Isla Santay.

\begin{tabular}{|c|c|}
\hline Propietarios & Nombre de la hacienda \\
\hline Jaime Nebot & La Puntilla \\
\hline Pedro Menéndez & Las Acacias \\
\hline Tomás Rolando & Pradera Grande \\
\hline Felipe de la Cuadra & Pradera Chica \\
\hline Guillermo Gilbert & La Florencia, \\
\hline Walter Guzmán & La Matilde \\
\hline Alfredo Guzmán & San Francisco. \\
\hline
\end{tabular}

Fuente: Elaboración propia.

En octubre de 1979, por decreto presidencial del extinto Jaime Roldós Aguilera, las tierras y propiedades de los hacendados fueron embargadas, pasando a pertenecer al Ministerio de Obras Públicas y Comunicaciones. Un año más tarde, la tenencia de la isla pasó al Banco Ecuatoriano de la Vivienda (BEV), institución estatal a la que se le atribuye la posesión hasta el día de hoy (Perrone, 2013).

Si bien es cierto, la isla Santay dejó de tener hacendados y posesión privada, las personas que vivían en el sitio firmaron con el BEV un convenio que los acreditaba como "guardias ad honorem", lo que les dio cierta legitimidad para asegurar su estadía (Perrone, 2013).

En el año 2000, por decreto del Ministerio de Desarrollo Urbano y Vivienda (MIDUVI), se establece un Comité Asesor para el desarrollo de la Isla Santay, el mismo que sugirió que una entidad debería hacerse cargo de los proyectos que pudieran realizarse en el lugar (Perrone, 2013). A partir del año 2001, se crea el "Fideicomiso Mercantil Isla Santay", que beneficiaba tanto al BEV como a la Fundación Malecón 2000 (creada a partir del proyecto de regeneración urbana del mismo nombre, que se ejecutó en Guayaquil), para que se elaboren y ejecuten proyectos de desarrollo ecológico, recreacionales y turísticos, para convertir a la isla en un parque ambiental y recreativo (Alcívar y Freire, 2017). El fideicomiso iba a estar vigente por 80 años. 
En el año 2007, el cantón Durán también reclamó sus derechos sobre la Isla, ya que jurisdiccionalmente pertenece al mismo. El gobierno del entonces presidente Rafael Correa dio por terminado el fideicomiso, pasando la isla al anterior propietario (el BEV) y su manejo a cargo del Ministerio del Ambiente (Perrone, 2013).

Hay que mencionar que, por su importancia ecológica y ambiental, en octubre del 2000 , la isla fue declarada, por la Convención Ramsar, como humedal de importancia internacional; y, posteriormente, en el 2010, fue declarada "Área Nacional de Recreación" (Alcívar y Freire, 2017), por lo que actualmente es parte del Sistema Nacional de Áreas Protegidas del estado ecuatoriano.

\section{El desarrollo turístico en Isla Santay}

Como se ha mencionado en párrafos anteriores, el turismo permite el sustento de muchas familias alrededor del mundo, algo que se hace palpable en el ámbito rural, ya que, en ocasiones, en ese sector las oportunidades del trabajo escasean. Esto lo reconoce la Organización Mundial de Turismo, al indicar que (Organización Mundial del Turismo, 2000):

a) El turismo puede generar nuevos puestos de trabajo, sobre todo a jóvenes, mujeres y grupos étnicos. Los puestos generados por la actividad turística pueden evitar la migración de jóvenes.

b) El desarrollo turístico puede motivar el establecimiento de nuevas empresas de turismo locales, por lo que podría generar un nuevo impulso económico en la zona donde se desarrolle.

c) Los beneficios del turismo pueden quedarse en la misma zona, sobre todo si las empresas o negocios que se establezcan pertenecen a la población local.

d) En general, las personas que empiezan a laborar en actividades turísticas se capacitan en diferentes aspectos: atención al cliente, ventas, administración de empresas y otras, lo que se traduce en un aumento de los conocimientos técnicos, que pueden ser transferidos a otras áreas.

e) El turismo necesita el desarrollo de una infraestructura adecuada, como: carreteras, agua potable, energía eléctrica, gestión de residuos o telecomunicaciones, lo que también beneficia a la población local.

f) La calidad ambiental también mejora, ya que los turistas prefieren ir a lugares limpios, ordenados y no contaminados. 
g) El turismo estimula la conciencia ambiental y un mayor respeto por las tradiciones culturales locales, así como a la protección -en caso de encontrarse- de sitios históricos y arqueológicos.

Por otro lado, se identifica, en la zona de estudio, que el turismo ha traído algunos beneficios: mejor accesibilidad, empleo relacionado a la actividad, participación comunitaria, mejoras en las viviendas familiares, utilización de energía limpia (solar), creación de espacios para alojamiento. Por supuesto, sin el impulso obtenido por la creación del puente peatonal de acceso, más las declaratorias de sitio Ramsar y área protegida, el nivel y calidad de vida dentro de la isla hubiera seguido sin mayor variación tal como existía desde el momento que el sitio comenzó a poblarse.

Asimismo, en el lugar puede identificarse elementos del turismo comunitario (Community based Tourism, - CBT en ingleés). En efecto, en este tipo de turismo “...es la propia comunidad local la que tiene el control y el desarrollo de la gestión turística..." (Muñoz, Ortega, Díaz, y López, 2017) y, por supuesto, la mayor cantidad de beneficios económicos generados por el turismo, se quedan dentro de la misma comunidad.

Las declaratorias de área protegida en la Isla Santay, que se mencionaron en párrafos anteriores, permiten también cumplir con una estrategia mucho más amplia de desarrollo sustentable, es decir, no solo se puede comprobar la protección ambiental, sino también el trabajo comunitario en el lugar, llegando a observarse algunos emprendimientos locales, sobre todo en lo referente a oferta de alimentación, donde los platos que se sirven se relacionan con la gastronomía típica de la costa ecuatoriana, lo que da como resultado que este tipo de turismo comunitario, de una u otra forma, “...permite mantener, proteger e incluso potenciar el patrimonio y los bienes culturales" (Oyarvide, Roldán, y Arias, 2016).

Es necesario indicar que el desarrollo de la actividad turística en la isla Santay se debió sobre todo a la construcción de un puente peatonal basculante -para el paso de los barcos por el río Guayas- y una pasarela, al interior de la isla. Con estos elementos construidos y en funcionamiento, la cantidad de visitas a la isla Santay aumentaron de 900 personas en 2012 a 717.818 en 2014 año en que se inauguró el puente peatonal (Díaz, Pérez, \& Ortega, 2017).

\section{Discapacidad, accesibilidad y sociedad}


El término 'discapacidad' presenta diversas interpretaciones dependiendo del paradigma desde el que se define y, debido a las diferencias de criterios, no hay acuerdo sobre su estimación, lo que hace que sea muy complicado comparar los datos estadísticos existentes y hablar con coherencia de la situación de las personas con discapacidad a escala internacional (Martínez, 2013).

La Organización Mundial de la Salud, en el "Informe Anual de la Discapacidad", entrega varios datos con respecto a este tema (WHO, 2011):

a) Más de mil millones de personas, en el mundo, viven con algún tipo de discapacidad, esto es, alrededor del $15 \%$ de la población mundial, lo que superó las expectativas de la misma Organización Mundial de la Salud, que, en los años 70's, indicaba que sería el $10 \%$.

b) El número de personas con discapacidad está creciendo en el mundo, debido a que la población está envejeciendo, al incremento de problemas crónicos de salud derivados de la discapacidad, como diabetes, enfermedades cardiovasculares y trastornos mentales.

c) La discapacidad es muy variada: tradicionalmente se la enfoca en personas en sillas de ruedas, ciegas o sordas; pero el espectro es mucho más amplio: desde personas autistas o con problemas mentales leves, hasta personas con graves problemas de salud que no pueden levantarse de una cama.

d) Las mujeres con discapacidad, además de las limitaciones propias de la condición física o mental que presenten, se enfrentan a una discriminación por su género.

e) A pesar de los graves problemas que se enfrentan muchas de las personas con discapacidad, sus cuidadores no siempre están bien remunerados y peor capacitados.

f) Existen graves actitudes negativas con respecto a las personas con discapacidad: muchos niños con alguna alteración física o mental no pueden acceder a una educación inclusiva y de calidad; mientras que muchos empleadores opinan que las personas con discapacidad son menos productivas que homólogos sin discapacidad.

g) En general, la mayoría de edificios no están adaptados para facilitar el acceso a personas con diferentes tipos y grados de discapacidad.

Así mismo, la Convención Sobre los Derechos de las Personas con Discapacidad indica lo siguiente sobre este grupo humano: 
Las personas con discapacidad incluyen a aquellas que tengan deficiencias físicas, mentales, intelectuales o sensoriales a largo plazo que, al interactuar con diversas barreras, puedan impedir su participación plena y efectiva en la sociedad, en igualdad de condiciones con las demás (ONU, 2006).

En la misma convención, se indica que los estados firmantes de la misma, se comprometen, entre otras cosas, a:

a) Adoptar medidas legislativas, administrativas y otras, a fin de que puedan cumplir con los derechos reconocidos en aquel documento.

b) Considerar en todas las políticas y programas que los estados promuevan, la protección y promoción de los derechos humanos de las personas con discapacidad.

c) Promover la investigación de nuevas tecnologías que se enfoquen en el diseño universal o la adaptación necesaria para el uso de diferentes elementos para personas con discapacidad.

d) Promover la formación profesional del personal que trabaja con personas con discapacidad, para que puedan prestar un servicio y asistencia de calidad.

\section{El turismo accesible}

Punto importante de análisis en el presente escrito es la relación entre la actividad turística y la accesibilidad, debido a que, como se verá más adelante, se explicará la experiencia de un grupo de personas con discapacidad física en este espacio protegido.

El turismo accesible "...es uno de los segmentos de mercado que mayor protagonismo ha cobrado en los últimos años..." (Fernández, 2009), por dos razones fundamentales: el número de personas con discapacidad que requieren de atractivos y servicios turísticos accesibles y la participación, cada vez mayor, de las personas con discapacidad en el mercado turístico (Fernández, 2014).

A pesar de esta afirmación, se hace necesario aclarar que el turismo accesible ha sido conceptualizado de forma reciente. Fue utilizado en 1989, por primera vez, en el llamado "Informe Baker", publicado en Inglaterra, donde se menciona a la accesibilidad “... a través de la supresión de barreras urbanísticas y arquitectónicas, así como a la integración social a través del turismo de las personas con algún tipo de discapacidad" (Jurado, 2014) . 
La Organización Mundial de Turismo, por su parte, en el Manual sobre turismo accesible para todos, señala que no existe un concepto consensuado sobre turismo accesible, "...debido a que es un concepto que ha experimentado una gran evolución durante los últimos diez años" (Organización Mundial del Turismo, 2016).

En la misma publicación, se hace una revisión cronológica de los diferentes conceptos que se han ido describiendo en el curso de los últimos 20 años (Organización Mundial del Turismo, 2016):

Tabla 2: Evolución del concepto de Turismo Accesible.

\begin{tabular}{|c|c|c|c|}
\hline Concepto & Fuente & Año & $\begin{array}{c}\text { Características del } \\
\text { concepto }\end{array}$ \\
\hline $\begin{array}{l}\text { “Conjunto de } \\
\text { actividades realizadas } \\
\text { durante el tiempo libre } \\
\text { dedicado al turismo } \\
\text { por personas con } \\
\text { capacidades } \\
\text { restringidas, que les } \\
\text { posibilita su plena } \\
\text { integración desde la } \\
\text { óptica funcional y } \\
\text { psicológica, } \\
\text { obteniendo la plena } \\
\text { satisfacción individual } \\
\text { y social” }\end{array}$ & $\begin{array}{l}\text { Grünewald, L. et al. (1996), } \\
\text { Turismo Accesible: } \\
\text { Experiencias y reflexiones } \\
\text { sobre accesibilidad, AMSCA } \\
\text { y Fundación Delia Lascano } \\
\text { de NAPP. }\end{array}$ & 1996 & $\begin{array}{l}\text { Concepto más enfocado } \\
\text { en ciertas discapacidad -a } \\
\text { pesar de parecer } \\
\text { abarcador- pues de cierta } \\
\text { forma excluye algunas } \\
\text { discapacidades } \\
\text { temporales }\end{array}$ \\
\hline $\begin{array}{l}\text { “Un proceso que } \\
\text { permite a las personas } \\
\text { con discapacidad y a } \\
\text { las personas mayores } \\
\text { funcionar de manera } \\
\text { independientemente, } \\
\text { en igualdad de } \\
\text { condiciones y con }\end{array}$ & $\begin{array}{l}\text { Darcy, S. (2006), Setting a } \\
\text { Research Agenda for } \\
\text { Accessible } \\
\begin{array}{l}\text { Sustainable Tourism, } \\
\text { Cooperative }\end{array} \\
\text { Centre, Gold Coast. }\end{array}$ & 2006 & $\begin{array}{l}\text { El concepto busca integrar } \\
\text { a las personas con } \\
\text { discapacidad mediante } \\
\text { productos o servicios } \\
\text { universales. }\end{array}$ \\
\hline
\end{tabular}




\begin{tabular}{|c|c|c|c|}
\hline $\begin{array}{l}\text { dignidad a través de la } \\
\text { prestación de } \\
\text { productos, servicios, y } \\
\text { entornos universales } \\
\text { en el turismo" }\end{array}$ & & & \\
\hline 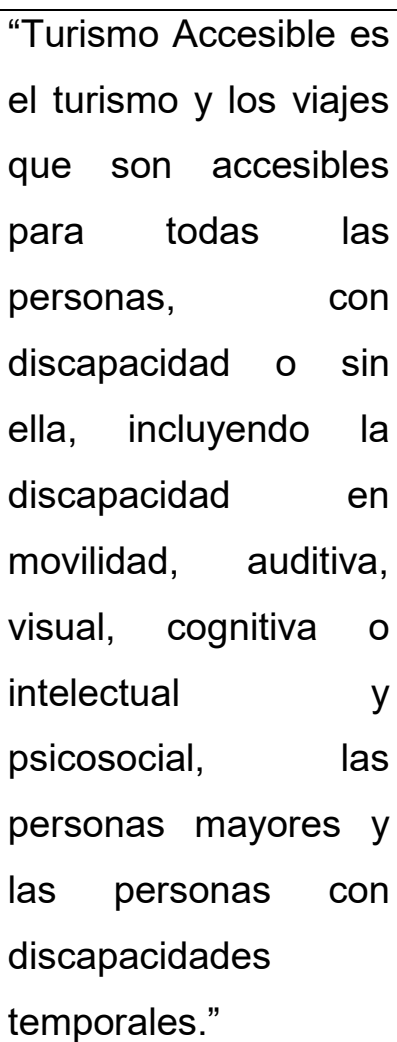 & $\begin{array}{l}\text { Economic and Social } \\
\text { Commission for Asia and the } \\
\text { Pacific (2009), Takayama } \\
\text { Declaration on the } \\
\text { Development } \\
\text { Communities-for-All in Asia } \\
\text { and the Pacific }\end{array}$ & 2009 & $\begin{array}{l}\text { El concepto ya define a la } \\
\text { accesibilidad como algo } \\
\text { para todas las personas, } \\
\text { con o sin discapacidad, } \\
\text { indicando cuáles son los } \\
\text { tipos de discapacidades, la } \\
\text { tercera edad y la } \\
\text { existencia } \\
\text { discapacidades } \\
\text { temporales }\end{array}$ \\
\hline $\begin{array}{l}\text { "Turismo Accesible es } \\
\text { el continuo empeño } \\
\text { por garantizar que los } \\
\text { destinos turísticos, } \\
\text { productos y servicios } \\
\text { sean accesibles para } \\
\text { todas las personas, } \\
\text { independientemente } \\
\text { de sus limitaciones } \\
\text { físicas, discapacidad o } \\
\text { edad. Esto incluye } \\
\text { lugares de interés } \\
\text { turístico de propiedad } \\
\text { pública y privada. Las }\end{array}$ & $\begin{array}{l}\text { Agnes, F. et al. (2010), } \\
\text { Accessible Tourism, VDM } \\
\text { Publishing. }\end{array}$ & 2010 & $\begin{array}{l}\text { El concepto se amplía } \\
\text { mucho más, al mencionar } \\
\text { a todas las personas con } \\
\text { discapacidad o sin ella, a } \\
\text { familias con niños } \\
\text { pequeños, personas } \\
\text { mayores y discapacidades } \\
\text { temporales. }\end{array}$ \\
\hline
\end{tabular}




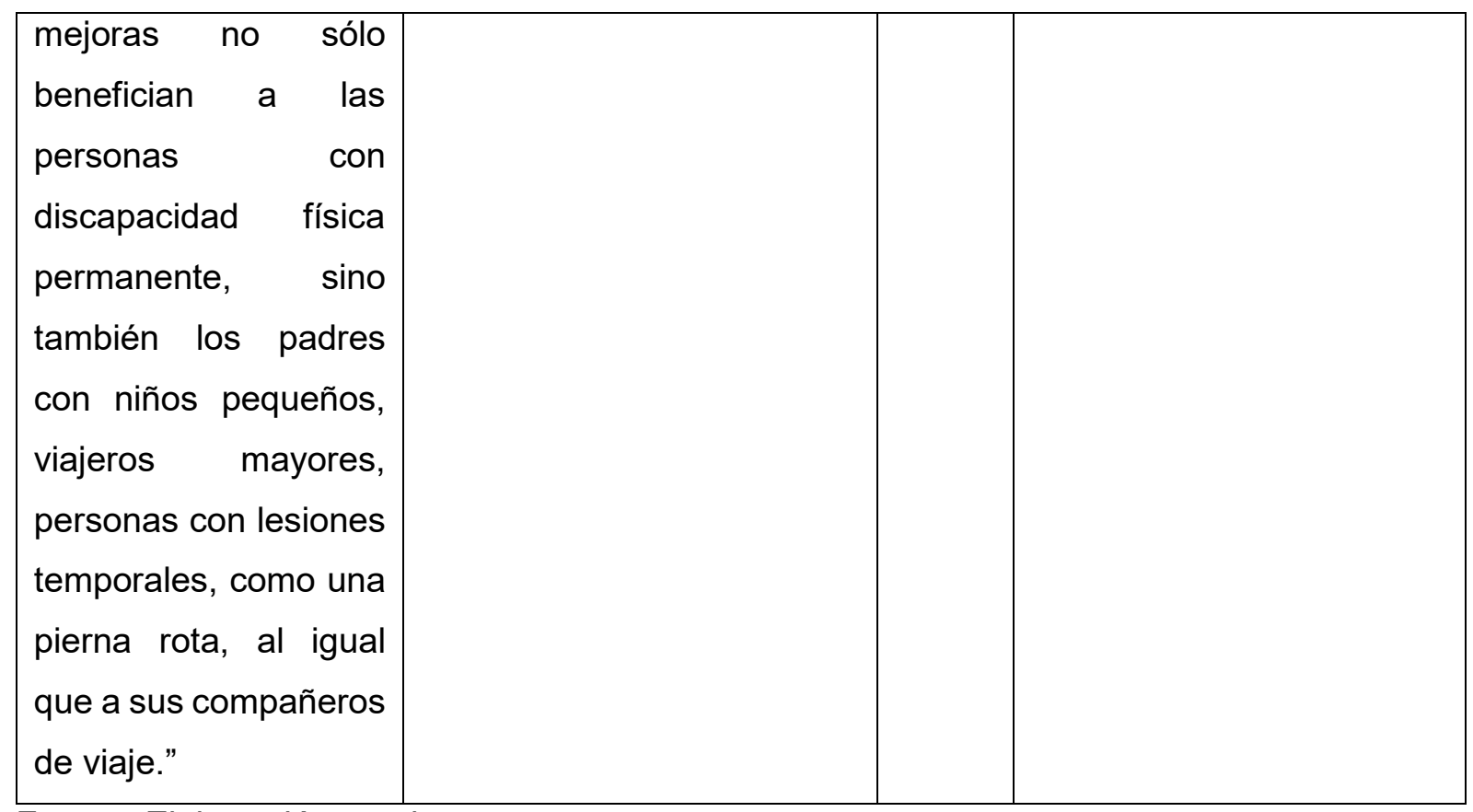

Fuente: Elaboración propia.

Como puede observarse en la tabla, el concepto de turismo accesible ha ido evolucionado, partiendo desde una idea enfocada solo en las personas con discapacidad -y muchas veces, solo la física- hasta llegar, en los actuales momentos, a involucrar a todas las personas y a otros sectores sensibles dentro de la sociedad, como familias con niños pequeños y personas de la tercera edad, sin olvidar a las personas con algún tipo de discapacidad temporal o con dificultades de movilidad, también temporal, como mujeres embarazadas, por ejemplo.

Ahora bien, con todas estas revisiones conceptuales, vale la pena destacar también las ideas relacionadas con el diseño universal, utilizado en Estados Unidos, o diseño para todos, de uso frecuente en Europa. Una aproximación más concreta a este concepto se lo encuentra en el Manual para un entorno accesible (Fernández, García, Juncá, Rojas, 2005), donde se explica que el diseño para todos o universal:

a) Elimina la idea que la accesibilidad solo se relaciona con la discapacidad física y las personas que la padecen, involucrando a los niños pequeños, ancianos, personas con baja estatura, personas con exceso de peso.

b) Evita la idea de un modelo único de persona que representa a la mayoría y para la que se diseña todo, pasando a ideas más universales de diseño.

c) Incluye el respeto a las minorías y respeto a las diferencias. 
d) Busca soluciones para todos, ya que todas las personas tienen derecho a usar y disfrutar el entorno en igualdad de condiciones.

e) Sugiere que el alcance del término "diseño" abarque más aspectos que la arquitectura y el urbanismo.

f) Precisa que el término "accesibilidad" sea mucho más amplio, ya que, si una persona se desplaza, cualquiera que sea su condición física, es para realizar una actividad.

A continuación, y con base a todo lo descrito en este breve marco teórico, se explicará la situación de la Isla Santay, describiendo una salida recreativa con personas con discapacidad física, donde se buscará cotejar los conceptos vertidos en este escrito, describiendo la salida particular que se redactará.

\section{MATERIALES Y MÉTODOS}

La experiencia que se describirá en las siguientes partes del escrito se relacionan con una salida recreativa inclusiva organizada por la Fundación AndarEQ, conformada desde octubre del 2018 y con sede en Guayaquil, a la que el autor del presente trabajo pertenece como Secretario. Es necesario mencionar que uno de los ejes de acción de la Fundación es "Ocio y recreación inclusiva", por lo que ha realizado, desde diciembre del 2018, diversos recorridos, sobre todo en el centro histórico de Guayaquil, dirigido a personas con discapacidad visual, auditiva y física, ya que se ha identificado que estos grupos sociales no siempre tienen oportunidad de disfrutar de espacios recreativos con libertad y seguridad.

Para la experiencia que se relatará, se invitó a 11 personas de la Asociación de Hemipléjicos, Parapléjicos y Cuadripléjicos y Amputados del Guayas (ASOPLEJICA), a un recorrido recreativo en la Isla Santay, como una forma de evaluar, para el grupo de personas con discapacidad física, el servicio y las instalaciones del sitio mencionado.

Antes y después del recorrido, se realizaron dos encuestas a las personas invitadas, para conocer sus características de salud y expectativas, antes del recorrido, y luego del mismo, para comprender sus opiniones luego de la experiencia.

Hay que mencionar que esta es una experiencia muy pequeña relacionada con la accesibilidad, el turismo y el uso de un espacio natural en la ciudad de Guayaquil, el mismo que cumple con ciertas ventajas para el traslado y disfrute del mismo. No se espera, de la 
descripción a realizar, obtener datos que puedan interpretarse como la opinión de todas las personas con discapacidad física, ni tratará de obtener conclusiones generales, sino muy puntuales relacionadas a la salida recreativa que se explicará.

\section{RESULTADOS Y DISCUSIÓN}

La salida fue realizada el día 6 de julio del 2019, a partir de las 09h30. Se inició, como se explicó anteriormente, con la bienvenida y la primera encuesta del día, para conocer las características del grupo. En este sentido, algunas de las preguntas y respuestas fueron las siguientes:

\section{Primera encuesta previa a la salida}

\section{Tipo de discapacidad}

Tabla 3. Tipo de discapacidad

\begin{tabular}{|l|l|l|}
\hline Indicadores & Frecuencia & Porcentaje \\
\hline Congénita & 11 & $100 \%$ \\
\hline Adquirida & 0 & 0 \\
\hline
\end{tabular}

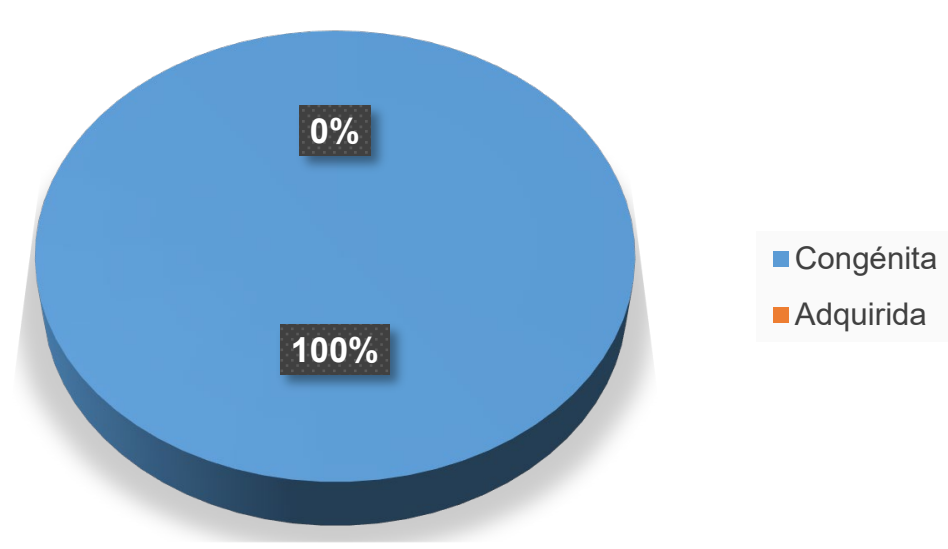

Gráfico 1. Tipo de discapacidad

El total de las personas invitadas presentaron una discapacidad adquirida, es decir, la obtuvieron durante el transcurso de su vida. La siguiente pregunta buscó indagar como fue adquirida esa discapacidad.

\section{Diagnóstico médico de la discapacidad}


Tabla 4. Diagnóstico médico de la discapacidad

\begin{tabular}{|c|c|c|}
\hline Indicadores & Frecuencia & Porcentaje \\
\hline Secuela de poliomelitis & 7 & $64 \%$ \\
\hline ACV & 1 & $9 \%$ \\
\hline Accidente & 1 & $9 \%$ \\
\hline Paraplejia & 2 & $18 \%$ \\
\hline Total & 11 & $100 \%$ \\
\hline
\end{tabular}

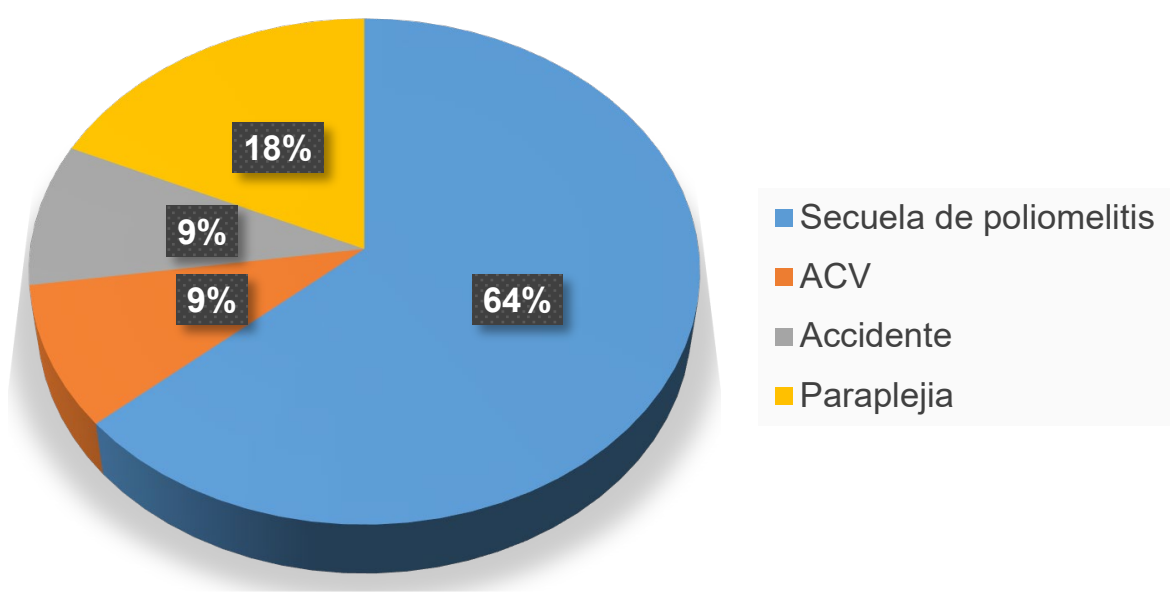

Gráfico 2. Diagnóstico de la discapacidad

Fuente: Elaboración propia.

Siete de las 11 personas entrevistadas, que corresponde al $64 \%$ del grupo, sufren su discapacidad como consecuencia de la poliomelitis. A pesar que en Ecuador la vacunación contra esta enfermedad es gratuita para niños entre 0 y 2 años, los padres no siempre consiguen vacunar a sus hijos, mientras que, en otros casos, la vacuna no fue de utilidad. No se indagó si los participantes fueron vacunados o no.

\section{Uso de ayudas para andar}

Tabla 5. Uso de ayudas para andar

\begin{tabular}{|c|c|c|}
\hline Indicadores & Frecuencia & Porcentaje \\
\hline Silla de ruedas & 9 & $82 \%$ \\
\hline Bastón & 2 & $18 \%$ \\
\hline Total & 11 & $100 \%$ \\
\hline
\end{tabular}




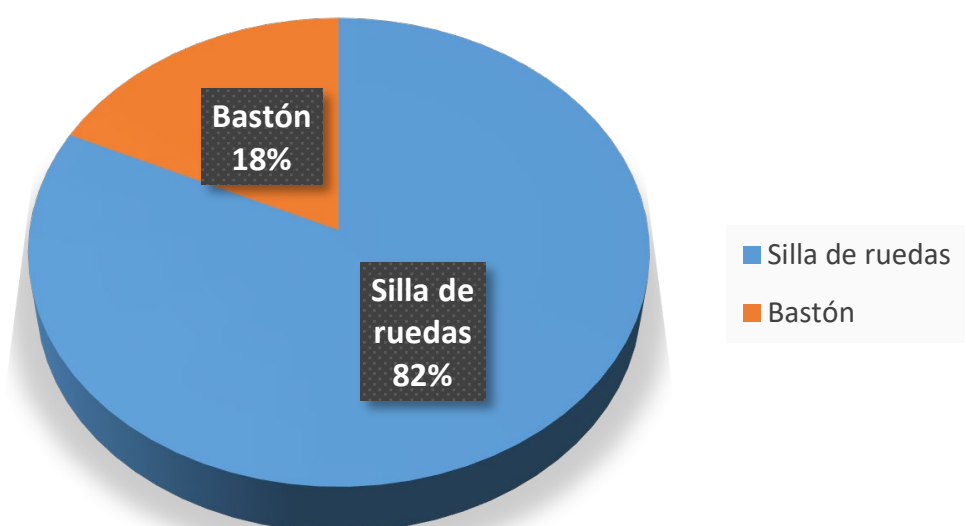

Gráfico 3. Uso de ayudas para andar

Fuente: Elaboración propia.

El $82 \%$ de los participantes (9 personas), se movilizaban en sillas de ruedas, lo que hace tomar mayor importancia en que los espacios y el camino a utilizar, dentro del área protegida, estén adaptados, tanto para este tipo de ayudas para el andar, como para cualquier persona que necesite desplazarse, para cumplir con uno de los postulados del diseño universal.

\section{Utilización de sonda vesical}

Tabla 6. Utilización de sonda vesical

\begin{tabular}{|c|c|c|}
\hline Indicadores & Frecuencia & Porcentaje \\
\hline $\mathrm{Si}$ & 9 & $82 \%$ \\
\hline $\mathrm{No}$ & 2 & $18 \%$ \\
\hline Total & 11 & $100 \%$ \\
\hline
\end{tabular}

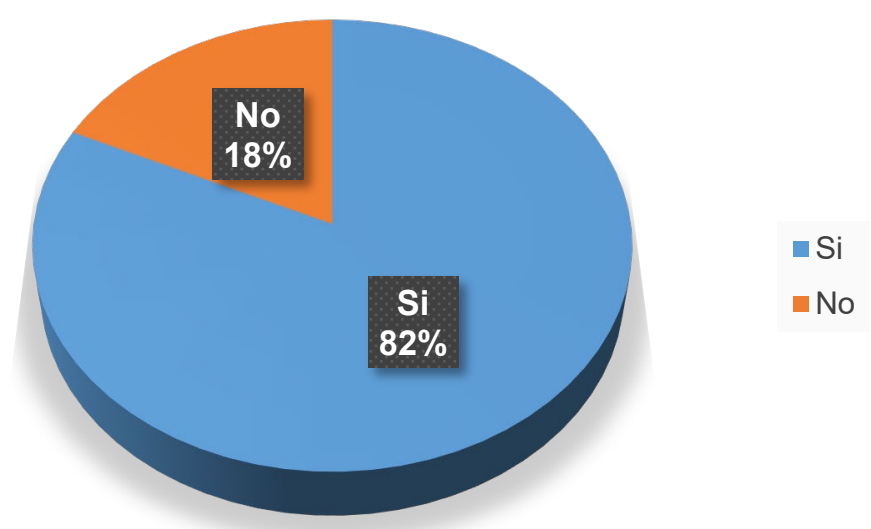

Gráfico 4. Utilización de sonda vesical

Fuente: Elaboración propia. 
Esta pregunta se relacionó con la calidad de vida y la comodidad de los participantes al momento de realizar la salida recreativa. Como puede observarse en el gráfico, el $82 \%$ de los participantes, esto es, nueve personas, no utilizan una sonda vesical. Sin embargo, era necesario conocer cuántos casos si la utilizaban a fin de poder tomar las medidas del caso, ya que esta sonda debe cambiarse cada cierto tiempo. En el caso de las dos personas que la utilizaban dentro del grupo, uno de ellos realizaba el vaciado de la sonda cada dos horas, y la otra persona, cada cuatro horas.

\section{Segunda encuesta post evento}

\section{Seguridad en la actividad}

Tabla 7. Seguridad durante la actividad

\begin{tabular}{|c|c|c|}
\hline Indicadores & Frecuencia & Porcentaje \\
\hline Muy bueno & 5 & $45 \%$ \\
\hline Excelente & 6 & $55 \%$ \\
\hline Total & 11 & $100 \%$ \\
\hline
\end{tabular}

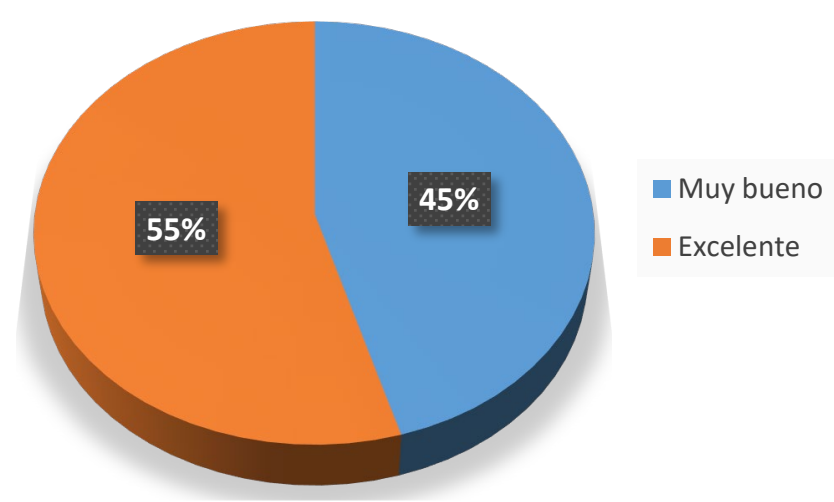

Gráfico 5. Seguridad durante la actividad

Fuente: Elaboración propia.

La seguridad del espacio visitado -Isla Santay- fue calificada entre excelente y muy bueno, ya que el sitio cuenta con una pasarela de cemento, que también actúa como puente basculante en cierta parte del recorrido, para permitir la navegabilidad en el río Guayas. A pesar que en la Isla la pasarela cambia de cemento a fibra, no existieron comentarios negativos con respecto a ese cambio.

\section{Atención y trato}


Tabla 8. Atención y trato durante el recorrido

\begin{tabular}{|c|c|c|}
\hline Indicadores & Frecuencia & Porcentaje \\
\hline Muy bueno & 2 & $18 \%$ \\
\hline Excelente & 9 & $82 \%$ \\
\hline Total & 11 & $100 \%$ \\
\hline
\end{tabular}

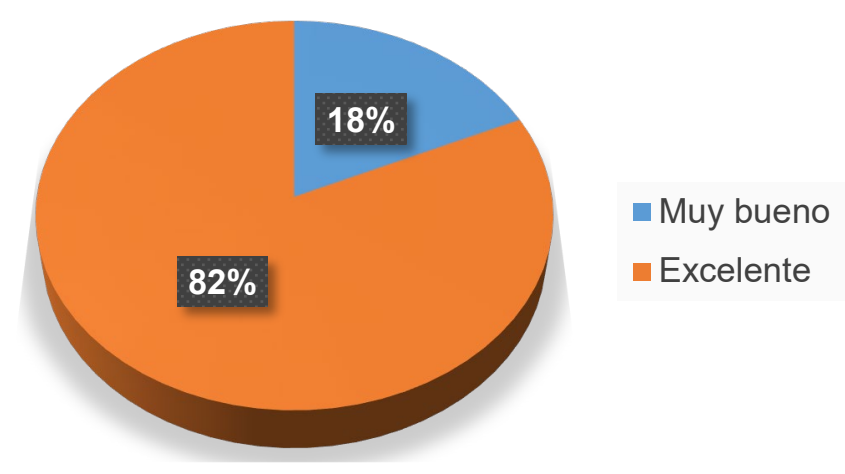

Gráfico 6. Atención y trato durante el recorrido

Fuente: Elaboración propia.

Uno de los puntos que debía tomarse en consideración era el referente a la atención y trato, tanto de los habitantes locales de la Isla Santay que son propietarios de emprendimientos sobre todo gastronómicos- como del personal del área protegida, que, a su vez, pertenecen al Ministerio del Ambiente. A pesar que hay muy poca costumbre de atender a grupos numerosos de personas con discapacidad -sea cual fuere la misma- durante el recorrido los asistentes expresaron su agradecimiento por la ayuda brindada y por la información recibida.

\section{Calidad de la información}

Tabla 9. Calidad de la información

\begin{tabular}{|c|c|c|}
\hline Indicadores & Frecuencia & Porcentaje \\
\hline Muy bueno & 2 & $18 \%$ \\
\hline Excelente & 9 & $82 \%$ \\
\hline Total & 11 & $100 \%$ \\
& & \\
\hline
\end{tabular}




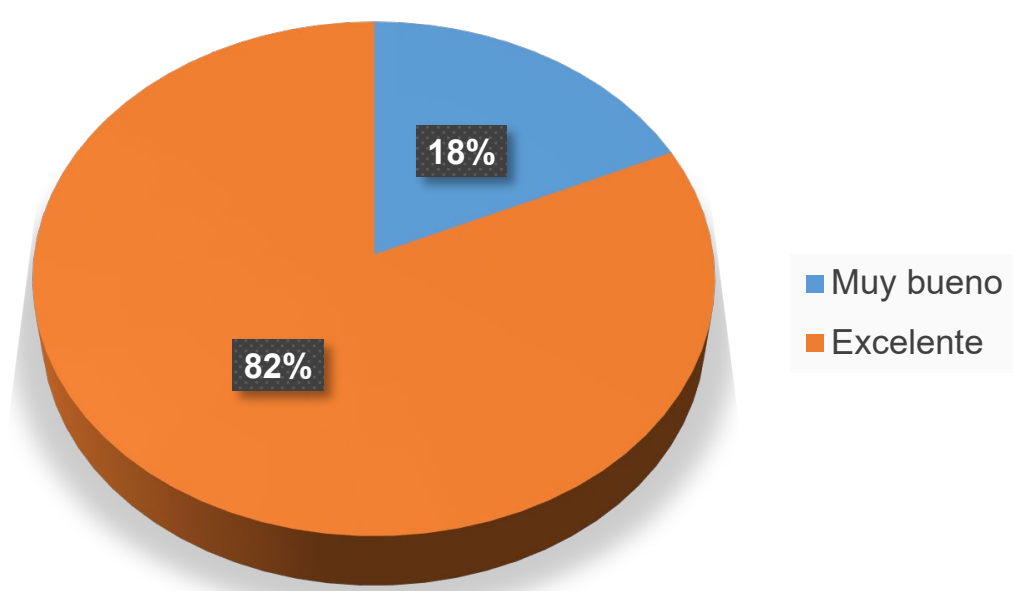

Gráfico 7: Calidad de la información

Fuente: Elaboración propia.

Con una calificación idéntica a la anterior, la calidad de la información fue calificada entre muy buena y excelente, sobre todo porque el guarda parques del área protegida que acompañó al grupo, supo transmitir, con mucha destreza, información científica y variada relacionada con el componente natural de la Isla Santay, información que, para el común de los habitantes de Guayaquil o de Durán, no es del todo conocida.

\section{Infraestructura y acceso}

Tabla 10. Infraestructura y acceso

\begin{tabular}{|c|c|c|}
\hline Indicadores & Frecuencia & Porcentaje \\
\hline Bueno & 3 & $27 \%$ \\
\hline Muy bueno & 5 & $46 \%$ \\
\hline Excelente & 3 & $27 \%$ \\
\hline Total & 11 & $100 \%$ \\
\hline
\end{tabular}




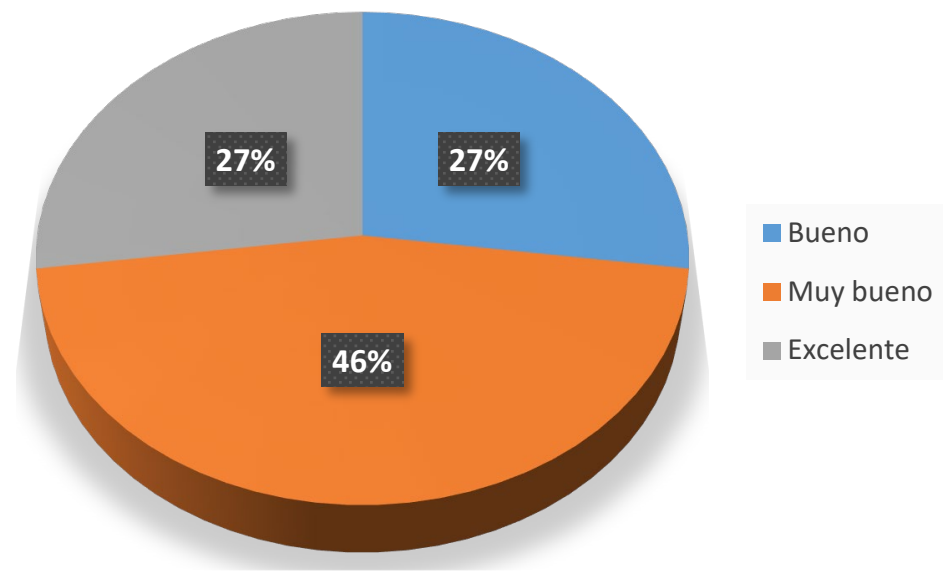

Gráfico 8: Acceso e infraestructura

Fuente: Elaboración propia.

Esta parte, del acceso e infraestructura de la Isla Santay, sobre todo en el puente que une la isla con Guayaquil y la pasarela para atravesarla y llegar a la comunidad (llamada Eco Aldea), fue calificada entre buena y muy buena, debido a que algunas partes de esa pasarela presentan agujeros, producto del paso de peatones, sobre todo en fines de semana. Entre los comentarios emitidos por los asistentes estaban que esos tramos deben ser reparados, ya que son un peligro constante, para personas con o sin discapacidad.

\section{CONCLUSIONES}

Luego del análisis conceptual y de los resultados de las dos breves encuestas, se puede concluir en este estudio de caso, que las personas con discapacidad deben tener las mismas oportunidades y facilidades para disfrutar de cualquier facilidad, en especial, las turísticas.

Sin embargo, al menos en Ecuador, y en concreto, a Guayaquil, se nota una falta de sensibilización ante el tema, ya que, en muchos casos de edificios o sitios públicos, muchas de las soluciones para la accesibilidad se refieren a la arquitectura, pero dejan de lado otros tipos de discapacidad, por lo que no existe una verdadera inclusión.

En el caso de la Isla Santay, como área protegida, puede decirse que presenta ciertas características adecuadas para la libre circulación para personas con discapacidad física pues es el caso que se analiza en el presente escrito- sobre todo a nivel de las estructuras de entrada y puente hacia la isla, pero la situación empieza a decaer cuando se utiliza la 
plataforma de fibro plástico colocada al interior, ya que, en la actualidad, deja ver muchos orificios en el piso y barandales caídos, lo que constituye un peligro para cualquier transeúnte.

Los niveles de seguridad no son los adecuados por las razones antes expuestas, además que, según se pudo comprobar, al interior de la Isla no existe un departamento médico para brindar los primeros auxilios, en caso de requerirlo cualquier persona, por lo que habría que trasladar al posible herido hacia la ciudad de Guayaquil.

A pesar de haber sido inaugurado en el 2014, el puente peatonal de cemento ya ha sufrido tres accidentes, en 2017, que fue el más grave, cayéndose 145 metros del total de la estructura, y dos en 2018, mucho más leves. Esto se debe a la fuerte marea que presenta el río Guayas, lo que hace que barcos con poca capacidad de maniobra (por reparaciones) no puedan evitar golpear el puente. Para el día del recorrido, algunas de las personas con discapacidad invitadas preguntaron sobre este tema, pero en el momento de la actividad, el puente se encontraba en buen estado y operando con normalidad.

No puede pensarse o suponerse que las personas con discapacidad física no pueden divertirse o explorar ciertos espacios. El grupo participante de la actividad arriba descrita disfrutó enormemente del paseo realizado, indicando que el hecho de utilizar una silla de ruedas, no significa que están condenados a no viajar. Por el contrario, el grupo participante pidió que este tipo de actividades pueda repetirse de forma constante.

Finalmente, cabe mencionar que la comunidad de la Isla Santay está consciente de su labor dentro del área protegida, por lo que trata siempre de brindar un servicio gastronómico de calidad a sus clientes, pues comprenden de la importancia de la actividad turística para su desarrollo, pero están conscientes que deben seguir mejorando y preparándose en diversos aspectos de la actividad turística.

\section{REFERENCIAS BIBLIOGRÁFICAS}

Alcívar, I., y Freire, Á. (2017). Análisis del Desarrollo Turístico Comunitario en el Área Nacional de Recreación Isla Santay - Ecuador. Ciencia y Tecnología, 2(December), 28-52. Retrieved from http://www.devuteg.com/revista/index.php/cienciaytecnologia/article/view/121 
Díaz, S., Pérez, J., y Ortega, M. (2017). Análisis De Las Motivaciones Hacia El Ecoturismo En Analysis of the Motivations Towards Ecotourism At Ecuador . a Case Study of Santay Island. Revista De Turismo Y Desarrollo Social, 10 (October), 1-19. Retrieved from file:///C:/Users/UNEMI.24-H102.000/Desktop/investigacion/islasantay.pdf

Fernández, J. de B.; García, J.; Juncà, J. A.; Rojas, C. de; S. G. J. J. (2005). Manual Para Un Entorno Accesible D O C U M E N T O S · 15/2005 Real Patronato Sobre Discapacidad (Quinta; con la colaboración de la F. A. Real Patronato sobre Discapacidad, ed.). Retrieved from www.cedd.net

Férnandez, M. T. (2014). Accesibilidad Para El Sector Turístico Accessible Tourism : Importance of Accessib-. (May).

Oyarvide, H. P., Roldán, A., y Arias, Y. (2016). Emprendimiento como factor del desarrollo turístico rural sostenible. Retos de La Dirección, 10(1), 71-93.

Hidalgo, J. A., Villafuerte, F., y Ortiz, C. (2015). Plan Estratégico De Desarrollo Turístico Sostenible Para EI Área Nacional De Recreación Isla Santay En Ecuador. Revista Observatorio de La Economía Latinoamericana. Retrieved from http://www.eumed.net/cursecon/ecolat/ec/2015/plan-estrategico.html

Jurado, J. M. (2014). El Turismo Accesible en Andalucía y Portugal. Cuadernos de Turismo, 31(33), 121-150. Retrieved from a=SPA

Martínez, B. (2013). Pobreza, discapacidad y derechos humanos. Revista Española De Discapacidad, 01(01), 9-32. https://doi.org/10.5569/2340-5104.01.01.01

Muñoz, G. A., Ortega, M., Díaz, S., y López, T. (2017). Turismo Comunitario Y Desarrollo Rural. Un Análisis de La Demanda Turística en La Isla Santay (Ecuador). PODIUM Sport, Leisure and Tourism Review, 6(4), 409-426. https://doi.org/10.5585/podium.v6i4.187

ONU. (2006). Convención sobre los derechos de las personas con discapacidad (ONU, 
ed.). New York: ONU.

Ordoñez, Y. M. (2017). Impact of Project " Generation and restoration of green areas for Guayaquil city "in population of Island Santay ". (July), 1813-1821.

Organización Mundial del Turismo. (2000). Guía para Administraciones Locales:

Desarrollo Turístico Sostenible (O. M. DEL TURISMO, ed.). Madrid:

ORGANIZACIÓN MUNDIAL DEL TURISMO.

Organización Mundial del Turismo. (2016). Manual sobre Turismo Accesible para Todos:

Principios, herramientas y buenas prácticas: Módulo V: Buenas prácticas de turismo accesible. In Organización Mundial del Turismo (Ed.), Manual sobre Turismo Accesible para Todos: Principios, herramientas y buenas prácticas: Módulo V: Buenas prácticas de turismo accesible (Primera). https://doi.org/10.18111/9789284416561

Pérez, J. C., Diaz, S., Muñoz, G. A., y Ortega, M. (2019). Satisfaction of the Tourist who Visits Santay Island (Ecuador). Community-Based Tourism is considered as an alternative to traditional tourism. Estudios y Perspectivas En Turismo, 28, 696714.

Perrone, G. N. (2013). La Isla Santay: entre la informalidad y la regeneración urbana de Guayaquil. In T. E. J. E. Bolívar (Ed.), Los lugares del hábitat y la inclusión (1st ed., p. 607). Quito: FLACSO.

WHO. (2011). Resumen de Informe mundial sobre la discapacidad. In Educación. Retrieved from http://who.int/disabilities/world_report/2011/summary_es.pdf 